\title{
Comment on Machida-Namiki-Araki Theory
}

\author{
M.M. Yanase \\ Sophia University, Tokyo
}

\begin{abstract}
Recent paper by S. Machida and T. Namiki proposed a very interesting idea of the generalization of quantum mechanios to macroscopic systems. Using this formalism, they have shown that the so-called "reduction of wave packet" in measuring process is explained without having the observer outside the measuring apparatus in the theory itself.

H. Araki proposed a framework of general theory of physics, which is in accord with the idea of Machida and Namiki. Following these ideas, together with the experimental evidence of Bell's inequalities, we have now a strong feeling that the nature has in principle a structure which allows us to formulate the physical theory which is general enough to include hitherto existing theories both in microscopic and macroscopic scales. Some fundamental problems on these ideas are discussed.
\end{abstract}

\section{Introduction}

Recently S. Machida and T. Namiki proposed a new theory of measurement in quantum mechanics.1) They have shown that the so-called reduction of wave packet by the observation can be described without having an "observer" outside the measuring apparatus.

They carefully examined many hitherto existing controversies and explained reasons of ambiguities and/or mistakes from their point of view.

The present author thinks that the proposal of Machida and Namiki is a remarkable contribution to the solution of difficult problems of quantum mechanical measurement process.

Moreover, the possibility of application of their formalism and the impact on epistemological, philosophical discussions on modern physics will be profound and far reaching.

On the other hand, recent reports on experimental research on Bell's inequalities') show that, the predictions following the theory based on any kind of hidden parameters to replace quantum mechanics get negative results, whereas the prsent theory of quantum mechanics gives the positive ones. From these data, we consider that the microscopic systems can be described only by quantum mechanics, judging from our present knowledge.

On accord with the idea of Machida and Namiki, H. Araki had developed a more general theory of physics, which is just like one mentioned above. Actually it was already proposed some 18 years ago by himself. ${ }^{3)}$ Historically speaking, what 
J. von Neumann described in his famous book: "Die Mathematische Grundlagen der Quantenmechanik, (1932)", ${ }^{4)}$ especially in chapters IV and V, might have aimed at the same idea as that of Araki. Neverthless, it was not possible to formulate such a theory as long as the problem of measurement was not solved consistently.

Now that Machida-Namiki-Araki theory, together with the experimental evidence on Bell's inequalities, give rise to think honestly on the general framework of physcis.

\section{Observer is not necessary}

Since the beginning of the development of quantum mechanics, especially after the detailed description of the theory of measurement in quantum mechanics by $J$. von Neumann, the problem of observer was discussed again and again by many authors. ${ }^{5)}$

J. von Neumann showed that, if we want to measure quantum mechanical quantities, it is necessary to have an observer, outside the measuring apparatus. The interaction between the object to be measured and the measuring apparatus transfers information on a quantity to be measured from the object to the measuring apparatus. However, the measurement is not accomplished, unless the observer "looks at" the apparatus and let the superposed state of the apparatus reduce to one of its eigenstates. This "looking at" the apparatus, which is called "reduction of wave packet" cannot be described by Schrödinger equation.

This strange dichotomy, and the necessity of introduction of an "observer" into the theory of measurement have been the rernel of the discussion. Most drastic expression of this dichotomy is the famous "Schrödinger's cat paradox". From the common sense point of view, it is very difficult to imagine that the states of macroscopic systems like those of a cat can form a superposition in quantum mechanical sense, and be reduced to one of the eigenstates of an operator to be measured, only through "looking at" the system by an "observer". Moreover, according to J. von Neumann, the cut between the object and the measuring apparatus can be shifted arbitrarily to the body of the observer, until the extreme point, where only the "consciousness" of the observer is left for the measuring apparatus.

To avoid this kind of difficulty, many interpretations, theories or opinions have been proposed.

The most popular interpretation is so-called Copenhangen interpretation, by Bohr, Heisenberg and their followers. They claim that the cut between the object and the apparatus should not be moved, and the object itself obeys quantum mechanics, whereas the apparatus follow classical mechanics. The uncontrollable interaction between them is the cause of the uncertainty relation: 
This rather ad hoc and practical interpretation made Quantum mechanics free from the difficult discussion on the measurement, and made it possible for the straightforward development of the theory. The weak point of this interpretation is the lack of logical consistency, consequently the lack of exact formulation.

Another group of authors tried to take the cut away from the theory. One subgroup of this approach claims so-called "hidden variable" theory. They try to re-formulate quantum mechanics in such a way that the statistical character of the theory is replaced by a deterministic one, even for microscopic systems.

This approach has consistency in so far as the entire world is described by classical causal relations. However, recent research on Bell's inequalities and their experimental tests show that hidden variable theory, at least local hidden variable theory, would not work ${ }^{2}$.

Another subgroup of this approach tries to move the cut towards the measuring apparatus all the way, so that the "observer" will not be necessary any more.

Various theories based on irreversible thermodynamics belong to this approach. They try to describe the measurement by irreversible process of the measuring apparatus. They are attractive because of detailed description of measuring process and suppression of vague concept like "consciousness" of the observer from the theory. At the same time, according to Wigner and Fine", these formalisms can not be consistent with quantum mechanics.

Theory of Machida and Namiki ${ }^{1)}$ belong to the last subgroup with fundamental differences from irreversible process approach. They also claim that the reduction of wave packet is accomplished without any observer outside the system. However, this reduction of wave packet is not the result of thermodynamical irreversible process of the apparatus. The cause of the reduction is the macroscopic character of the apparatus. They put the following conditions for the characteristics of the macroscopic apparatus.

I) The duration of measuring interaction between the object and the apparatus is very long in microscopic scale, but very short in macroscopic scale.

II) The measuring apparatus has macroscopic scale and cannot be described by a ray in a single Hilbert space, but should be described by a mean of rays of continuously many Hilbert spaces.

Using these conditions, and Riemann-Lebegue Lemma, they have shown that the reduction of wave packet occurs by the averaging process of time duration in microscopic scale for the object, and the multi-Hilbert spaces formalism for the macroscopic measuring apparatus.

Because of this new formalism, the theory is not contradictory to WignerFine theorem.

H. $\mathrm{Araki}^{3)}$ gave a precise mathematical description of multi-Hilbert space 
formalism, proposed by Machida and Namiki. He named it the continuous superselection rule, because it is a natural generalization of the discrete superselection rule by Wick, Wightman and Wigner to the continuous case.

The next problem is whether this formalism can replace hitherto existing physical theory for macroscopic systems. Unless it is done, the theory of MachidaNamiki and Araki (MNA theory for the brevity) remains only as an ad hoc solution for the problem of the measurement in quantum mechanics.

This problem will be discussed in the following section.

\section{Towards a general theory of Physcis}

H. Araki, in his article ${ }^{3}$ ) which is cited before, gave a positive proposal to the problem of the last section. He claims that the formalism of continuous superselection rule with a set of classical observables in his sense is equivalent to classical mechanics, if we add a proper dynamics to the formalism.

Following his description, we may say that both quantum mechanics and classical mechanics are described by the same framework which includes descrete and continuous superselection rule. Ordinary quantum mechanics is described by a single Hilbert space formalism, whereas discrete superselection rule by Wick, Wightman and Wigner, is described by a direct sum of multi-Hilbert space formalism, and finally, classical mechanics is described by a direct integral of continuously many Hilbert space formalism. Actually general mathematical tool for this argument was already prepared by many authors like Segal, Haag, Kastler and Araki using C*-Algebra).

To accomplish this equivalence, however, it is necessary to modify the definition of a concept in macroscopic systems.

In describing macroscopic systems; MNA introduced a direct integral $L$ of continuously many Hilbert spaces $L_{\lambda}$ for that purpose. A macroscopic system is described by vectors $f$, whose inner product is defined by

$$
\left(f_{1}, f_{2}\right)=\int\left(f_{1}(\lambda), f_{2}(\lambda)\right)_{\lambda} d \mu(\lambda) \text {, where }(,)_{\lambda} \text { denotes the inner product in } L \text {. }
$$

As Machida and Namiki explain in their second article (which will be written as M.N.-II, whereas their first article will be written as M.N.-I), this description of a macroscopic system is actually a mean of many vectors in $\mathrm{L}$, which can not be distinguished by macroscopic observables, but can be distinguished only by microscopic observables. H. Araki considers these macroscopic observables as classical observables and call this formalism the continuous superselection rule as mentioned before.

This way of identifying system or ensemble of systems is different from what has been in practice in physical theory. Usual way of identifying system in physics is expressed e.g. by a definite number of particles, $N_{0}$. If the number of particles of 
another system is $\mathrm{No}+I$, it belongs to a different system. But in MNA theory, a mean of many systems of different particle numbers is described as a single system and denoted by, e.g. $f_{1}$ in $L$.

This way of describing a mean of systems is not the same as probabilistic description of systems in statistical mechanics, because we do not consider an ensemble of systems, whose number of particles are not known, but that the fluctuation of numbers of particles is disregarded.

Considering this situation, I conclude that the logical way of describing a concept of a macroscopic system should be different from usual say. Discussion on this problem will follow in the next section.

\section{Fuzzy concept.}

The way of describing a concept like one just mentioned is known in system engineering since 1960, initiated by Zadeh. They are called fuzzy concepts and the logical theory based on fuzzy concepts is called fuzzy logic.

The detailed discussions on fuzzy logic and fuzzy concepts are found in numerous publciations ${ }^{6}$.

I limit myself to consider the characteristics of fuzzy concepts as far as it is appropriate for the discussion of our problems.

It should be noticed first that the fuzziness does not exist in MNA-theory as long as they consider microscopic systems, the fuzzyness comes into the theory when we consider macroscopic systems from the general point of view of the theory.

As mentioned before, in $\mathrm{MN}-\mathrm{II}^{1)}$, a macroscopic system $f$ is expressed by a function of $D, f=f(D), D$ being microscopic variables, which can not be specified exactly in macroscopic scale, and the integral $f=\int f(D) d \mu(D)$ is considered to be a macroscopic state identified the same one.

This is precisely a way of defining a system by a fuzzy concept. It may be noted that the normalization factor $\int d \mu(D)=I$ should be in the denominator, so that $f$ is a mean of various states $f(D)$ over the whole range.

In fuzzy concept theory, developed by Zadeh and others, $f(D)$ is called the membership function.

This is a new way in physical theory of looking at the macroscopic system and constructing a logical structure on the whole problem.

As mentioned elsewhere ${ }^{7}$, the idea of fuzzy concepts and fuzzy logic is a very natural generalization of formal logic and actually it is almost always used in our daily life.

I consider, therefore, it is necessary to introduce fuzzy concepts into MNA theory, and make further investigation along this idea. I even hope that this kind of investigation may give some light on the theory of fuzzy logic itself. 


\section{Further physical problems.}

There still remain, of course, several problems to be investigated for the development of MNA-theory.

As for the relativistic formalism of the theory, including electro-magnetic phenomena, it may not be too difficult to do it, because $\mathrm{C}^{*}$-Algebra formalism is general enough to include special relativity.

Whether it is possible to include the general relativity, I do not know. The only point which I can make is that if the general gauge theory is successful to describe the general relativity as well as the particle theory, it is a challenging problem, to try to incorporate it into MNA-theory.

It is also important to investigate how to consider statistical phenomena, especially those of low temperature from the view point of the new theory.

\section{Philosophical discussions}

We should be very careful to discuss on the philosophical implications, when a new physical theory is invented.

The discussions will be fruitless, unless the new theory itself is not established as physical theory.

Furthermore, it is important to know about the methodological implications for the discussion to be fruitful. Ignorance on these implications often make discussions quite confusing.

Until recently philosophical discussions on the problem of measurement in physics has been centered on that of quantum mechanics. MNA-theory gives a light on this problem giving a consistent scheme to discribe the measuring process. The result is, as explained above, that we do not need an outside "observer" for the theory.

Moreover, we now know that macroscopic system can be discribed by the MNA-theory, which is equivalent to classical mechanics.

It looks like that the problem of the measurement of physcis in general is solved at last. Is it true? I do not think so. I think that now for the first time the real philosophical or more precisely, the epistemological problem of measurement in Physics in general can be discussed honestly.

Unless the problem of the measurement was not solved within the framework of physics, it was not possible to discuss the same problem philosophically.

This has been the conviction of the author, and it was the reason why he first tried to work on the problems within the framework of physics.

In classical physics, it is tacitely presumed that we can measure physical quantities of a given system from outside, without changing the systems itself.

In quantem mechanics, it is even more ture, if we measure a physical quantity, when the system is in one of its eigenstates. 
MNA-theory assured that these two situations are equivalent in macroscopic systems, and for microscopic systems we can have an interaction between the microscopic object and the macroscopic apparatus, such that the microscopic quantities can be measured through the macroscopic apparatus, consistent with the so-called reduction of wave packet in the microscopic object.

Now we ask, what is the mechanism of the measurement on a macroscopic or, equivalently, a classical system by an observer ?

This is simply presupposed in physics, but this is the real epistemological problem.

Philosophically, the attitude for such a presupposition is nothing but naive realism.

The problem is, therefore, whether one can be satisfied as naive realist or not. If not, then the problem should be further investigated.

The author should express his sincere gratitude to professors Machida, Namiki and Araki for their most stimulating discussions.

His gratitude extends also to participants of the symposium on this problem held in the 1980 annual meating of the Japan Association of Philosophy of Science, as well as the study meeting at Yukawa Hall on the same problem in 1980.

\section{References}

1) S. Machida and T. Namiki, Progress of Theor. Phys. 63 (1980) 1457 \& 1833.

2) J.F. Clauser and A. Shimony, Bell's Theorem: Experimental Tests and Implications, Report of Progress in Physics 41, 1881 (1978), Bristol.

3) H. Araki, Progress of Theor. Phys. 64 (1980) 719.

4) J.v. Neumann, Die Mathematische Grundlagen der Quantenmechanik, (1932).

5) B. d'Espagnat, Conceptual Foundations of Quantum Mechanics. 2nd ed. (1976).

6) e.g. L.A. Zadeh, King-sun Fu, Kokichi Tanaka, Masamichi Shimura ed., Fuzzy Sets and Their Applications to Cognitive and Decision Process (1975).

7) M.M. Yanase, Ann. Jap. Assoc. Phil. Sci. 5225 (1980). 